\title{
A Theoretical Framework to Improve the Quality of Manually Acquired Data
}

\author{
Tom Haegemans ${ }^{\mathrm{a}, *}$, Monique Snoeck ${ }^{\mathrm{a}}$, Wilfried Lemahieu ${ }^{\mathrm{a}}$ \\ ${ }^{a}$ KU Leuven, Department of Decision Sciences and Information Management, Naamsestraat 69, B-3000 \\ Leuven, Belgium
}

\begin{abstract}
We present a framework for organisations to prevent errors in data entry. It states that data entry errors can be prevented by a strong intention of data producers to enter data correctly and by a high task-technology fit. Two empirical studies support the framework and demonstrate that a high task-technology fit is relatively more important than the data producers' intention. The framework refines the theory of planned behaviour, and extends the explanatory domain of the task-technology fit construct. The empirical evidence underlines the importance of the task-technology fit construct, an often-neglected construct in information systems research.
\end{abstract}

Keywords: data quality, data entry, manually acquired data, information quality

\section{Acknowledgement}

We would like to thank KBC Group NV for their financial and operational support.

\section{Declaration of Interest}

The first author was funded by the organisation at which one of the empirical studies took place (KBC Group NV).

\footnotetext{
*Corresponding author

Email addresses: tom.haegemans@kuleuven.be (Tom Haegemans), monique.snoeck@kuleuven. be (Monique Snoeck), wilfried.lemahieu@kuleuven.be (Wilfried Lemahieu)
} 


\title{
A Theoretical Framework to Improve the Quality of Manually Acquired Data
}

\begin{abstract}
We present a framework for organisations to prevent errors in data entry. It states that data entry errors can be prevented by a strong intention of data producers to enter data correctly and by a high task-technology fit. Two empirical studies support the framework and demonstrate that a high task-technology fit is relatively more important than the data producers' intention. The framework refines the theory of planned behaviour, and extends the explanatory domain of the task-technology fit construct. The empirical evidence underlines the importance of the task-technology fit construct, an often-neglected construct in information systems research.
\end{abstract}

Keywords: data quality, data entry, manually acquired data, information quality 


\section{Introduction}

Most of the data stored by organisations were once captured manually by human data producers (Maydanchik, 2007). Often, organisations have a good reason to still rely on manual data acquisition by humans instead of automatic procedures. Sometimes, automatisation of manual input procedures is simply impossible because the technology to replace these procedures is not yet developed. For example, consider the manual registration into an information system of observations made by psychiatrists while listening to their patients. At this moment, it is impossible to automate this procedure because no sensors currently exist that can make the same observations and register them automatically into an information system. Other times, it is too expensive to automate the manual input procedures. For instance, the complex business processes in financial institutions resulting from a complex hierarchy of financial products prove very costly to automate (Dias et al., 2012).

A significant (and well-known) problem with this manually acquired data is that it is prone to errors. Examples of datasets reported to contain errors are financial datasets (Authors, 2016), medical datasets (Thiru et al., 2003; Arts et al., 2002; Goldhill and Sumner, 1998), agricultural datasets (Espetvedt et al., 2013), inventory datasets (DeHoratius and Raman, 2008) and criminal datasets (Laudon, 1986). When reported, the percentage of erroneous records ranges from 0 (Weiskopf and Weng, 2013) to as high as 65\% (DeHoratius and Raman, 2008)

Even a single error in a dataset can have far-reaching negative consequences such as affecting statistical results, possibly leading to wrong decisions (Barchard and Pace, 2011; Kozak et al., 2015). In addition, it is not hard to find specific situations where a data entry error caused serious issues. For example, in a financial institution, a data entry error made by a clerk enabled a client to spend 2.1 million dollars without being noticed (Moore v. R, 2016, par. 55). In another case, a client of a financial institution instead of a clerk, made an error. The client accidentally typed a wrong digit in an account number, causing the transfer of 100,000 dollars to an unknown person (Olsen, 2008). Also, the aviation sector is affected by data entry errors. Recently, the captain of an Airbus A330 mistakenly entered the wrong coordinates into the autopilot of a plane. Because the error remained unnoticed until after the plane took off, the plane headed in the wrong direction and had to land at a different airport (Josephs, 2016). In this same manner, some time ago, a typo in a computer terminal caused a major cloud service provider to go offline (Newton, 2017).

These examples are illustrative of the importance of error prevention in manual data entry. This can be achieved by eliminating or mitigating their root causes. However, to the best of our knowledge, a theoretical framework that can help organisations identify root causes of errors in manually acquired data is missing from the literature.

In response, we propose and empirically evaluate the 'Causes of Errors in Manually Acquired Data' (CEMAD)-framework. The CEMAD framework is formulated at the level of an individual human data producer and states that two factors cause errors in manual data entry: a weak intention of the data producer to enter data correctly, and/or a low fit between the technology, task and individual (task-technology fit or TTF). The framework is grounded in empirical observations from an exploratory case study, and informed by the 
theory of planned behaviour and TTF model, which were combined to resolve the theoretical shortcomings explained in Section 2.2.

Both the theory of planned behaviour and TTF model are supported by a plethora of empirical findings that could potentially justify the core hypotheses of the CEMAD framework and therefore would not require gathering additional empirical evidence. However, as will be explained in Section 3.2, additional empirical findings are not only required to justify the CEMAD framework but such findings would also be of interest to practitioners. For example, the CEMAD framework gives clear indications of possible data improvement actions (i.e. actions should improve the TTF and/or strengthen the data producers' intention to enter data correctly). For practitioners, having such indications is useful, but for them, it is equally, if not more, important to know which kind of actions potentially have the largest impact on the actual behaviour. Therefore, additional empirical evidence was gathered by two experimental studies testing the core hypotheses of the CEMAD framework and examining the effect size of each of the framework's factors.

The remainder of this manuscript is structured as follows. Section 2 introduces the two relevant theoretical frameworks: the theory of planned behaviour and the TTF model, and delineates their shortcomings in explaining errors in manually acquired data. Section 3 details the CEMAD framework and argues why its core hypotheses require additional empirical evidence. Section 4 provides empirical evidence resulting from a large-scale experiment for the hypotheses of the CEMAD framework. Section 5 demonstrates how the CEMAD framework can be applied in practice and provides additional empirical evidence for certain hypotheses of the CEMAD framework. Section 6 discusses the empirical results stemming from both the experiment and application. Section 7 delineates the practical implications of the framework. Section 8 concludes the research and lists opportunities for future research.

\section{Theory Development}

\subsection{Relevant Theoretical Models}

In previous work (i.e. Authors, 2016), we adopted a grounded theory approach to analyse the observations during an exploratory case study in a major Belgian financial institution. During this study, we verified two home loan attributes using exhibits from the paper archive to find errors, and conducted interviews and consulted the documentation of the home loan information system to identify the causes of the errors we found. We found that all identified errors could be best explained by a combination of the theory of planned behaviour and TTF model.

\subsubsection{The Theory of Planned Behaviour}

The theory of planned behaviour (Ajzen, 1991) is an established theory to explain human behaviour and is based on the theory of reasoned action. The theory (Figure 1) states that the behaviour of an individual can be explained by how much actual control this individual has in order to perform the behaviour together with the intention that the individual has to exert the behaviour (Fishbein and Ajzen, 2010). In its turn, the intention of the individual is determined by a combination of the attitude of the individual towards performing the 
behaviour, how the individual believes that others think about the behaviour and how much the individual perceives that he or she is in control of performing the behaviour (Ajzen, 1991). Sometimes, the attitude construct is decomposed in an instrumental and experiential part (Fishbein and Ajzen, 2010, p. 82). Instrumental attitude captures cognitive aspects of attitude like usefulness while experiential attitude captures affective dimensions like pleasantness.

The intention of individuals is closely related to their motivation: if an individual is motivated to perform a certain behaviour, s/he has a strong intention to perform the behaviour. In other words, the intention of an individual captures or 'indexes' his/her motivation (Sheeran, 2002, p. 2).

When applied in a data entry context, the theory of planned behaviour can be used to construct data quality improvement actions that target the psychosocial antecedents of intention (Murphy, 2009). For example, the instrumental attitude of data producers towards entering data correctly can be strengthened by providing these producers with information about why it is important to enter data correctly (Lee and Strong, 2003; Murphy, 2009; Authors, 2017), or by providing them with monetary rewards. Their experiential attitude towards the behaviour of entering data correctly can become more positive by, for instance, making the information system more easy to use. The subjective norm of the data producers can become more positive if, for example, their peers state that they also enter data correctly and that they find correctly entered data important. The perceived behavioural control can be increased by, for example, making the data entry task less complex.

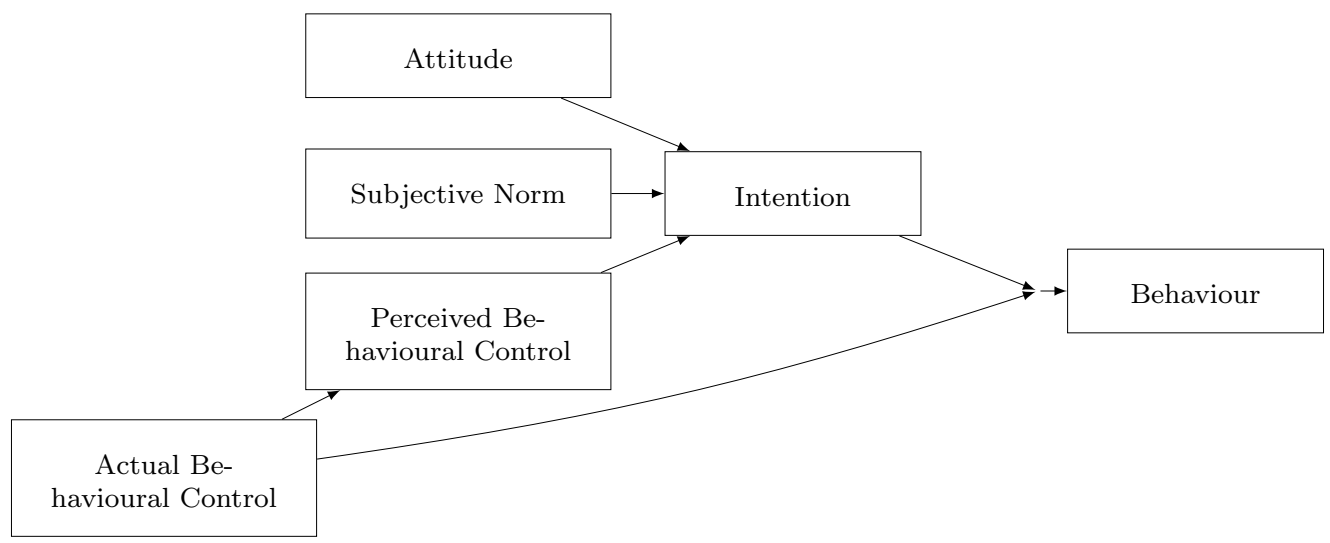

Figure 1: The Theory of Planned Behaviour (Ajzen, 1991)

\subsubsection{The Task-Technology Fit Model}

The task-technology fit (TTF) model, shown in Figure 2, states that the performance of an individual in executing a task is determined by the 'degree to which a technology assists an individual in performing his or her portfolio of tasks' (Goodhue and Thompson, 1995, p. 216) and by the 'behaviour [of the individual] of employing the technology in completing tasks' (Goodhue and Thompson, 1995, p. 218). In other words, the performance 
of an individual in executing a task is determined by the $\mathrm{TTF}^{1}$ and the utilisation of the technology (Goodhue and Thompson, 1995). In practice, this model is frequently used to explain how the errors of the output of an information system affect the performance of the task for which this output is used (see e.g. Goodhue, 1995, p. 1831). To the best of our knowledge, this model has not yet been applied in a data acquisition setting, while it could: the quality of data that a data producer entered can be considered as the performance (data quality) of this individual (data producer) in executing the (data entry) task. For example, in the context of manual data entry, a high TTF could mean that the structure of the data model (technology) fits the information structure of the real world (task) (Orr, 1998, p. 70), or that the design of the user interface (technology) reduces the short-term memory load of the data producers (individual abilities).

When the TTF construct is applied in the context of data entry, it is conceptually equal to the degree of actual behavioural control of the theory of planned behaviour. Indeed, when an information system (technology) fits the reality (task) and the individual abilities of the data producer, it offers the data producer the right opportunities and resources to enter data of high quality and thus provides him or her with a high degree of behavioural control.

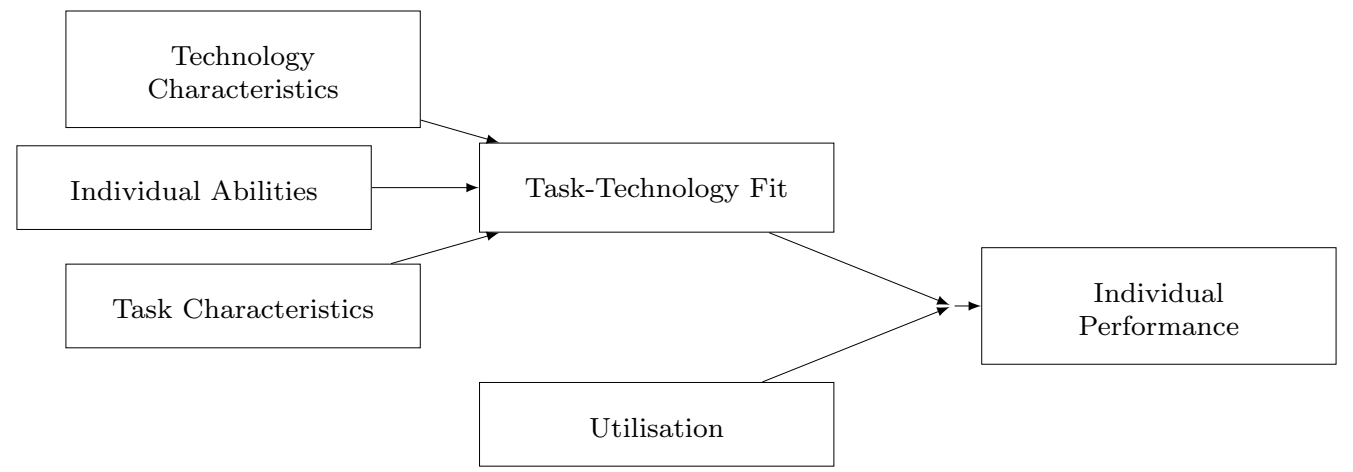

Figure 2: A Task-Technology Fit (TTF) Model (Goodhue and Thompson, 1995)

\subsection{Shortcomings of the Theoretical Models in Explaining Errors in Manually Acquired Data}

While both theories have their merits in clarifying the use of technology, they fall short when used with the aim of finding the root causes of errors in manually acquired data. To serve such a purpose, it is not only important that a theory is able to explain all or most of the errors in manually acquired data, but also that there is sufficient information available about the antecedents of its core constructs. Information about the antecedents of factors that explain errors in manually acquired data is important as it can serve as a basis for concrete data quality improvement guidelines.

The theory of planned behaviour was able to explain all errors in the manually acquired data in the exploratory case study (Authors, 2016, p. 15:6). However, the theory only

\footnotetext{
${ }^{1}$ Goodhue (2006) has indicated that 'a more accurate label for the construct might be task-individualtechnology fit, but the simpler TTF label is easier to use' (p. 190)
} 
delineates antecedents for one of its two core constructs: the individuals' intention to perform a certain kind of behaviour. The theory does not contain antecedents for the actual behavioural control construct.

Yet, in a manual data entry context, the data producers' resources could potentially have a significant impact on the correctness of the data which is even larger than that of their intention to enter data correctly. For example, in a recent replication of the theory of planned behaviour in a data entry context, we found that the statistical control variables related to actual behavioural control were more strongly correlated to errors in the entered data compared to the data producers' intention (Authors, 2018). As such, antecedents for the actual behavioural control construct would be of particular interest to organisations aiming to prevent errors in manually acquired data.

While each model has some shortcomings, they can easily be linked together as the TTF construct is a specialisation of the actual behavioural control construct, which are both conceptually equal in the case of manual data entry. Consequently, to explain errors in manually acquired data, we propose to combine both models as follows: replace the actual behavioural control construct of the theory of planned behaviour with the TTF construct of the TTF model. In the remainder of this paper, the resulting model will be called the 'Causes of Errors in Manually Acquired Data (CEMAD)-framework'. Compared to the theory of planned behaviour, the CEMAD framework is better able to explain errors in manually acquired data as the TTF construct specifies which resources individuals require to enter high quality data. By demonstrating the utility of the TTF construct to explain errors in the input of an information system, rather than to explain how errors in the output of an information system affect the performance of a task, we extend the explanatory domain of this construct.

\section{The Causes of Errors in Manually Acquired Data (CEMAD)-Framework}

\subsection{Research Model and Hypotheses}

The CEMAD framework, which is depicted in Figure 3, is empirically grounded in the results of an exploratory case study (i.e. Authors, 2016), and is informed by the TTF model and by the theory of planned behaviour. It posits that errors in manually acquired data can be explained by a low intention of the data producers to enter data correctly, by a poor TTF, or by a combination of those two factors. In this section, we will present the relevant constructs of both theories as part of the CEMAD framework, tailor the definitions of these constructs to the manual data acquisition context, and formulate hypotheses that are empirically verifiable.

Errors in Manual Data Entry. An error in manual data entry is defined as a situation where the data producer did not enter the correct registration for an attribute of a real-world object that he or she observed. These errors in manually entered data can be interpreted as the data producer having a low individual performance.

At the same time, this construct is related to data quality: an error in manual data entry results in a deficiency in the quality of this data (Wand and Wang, 1996, p. 89). 


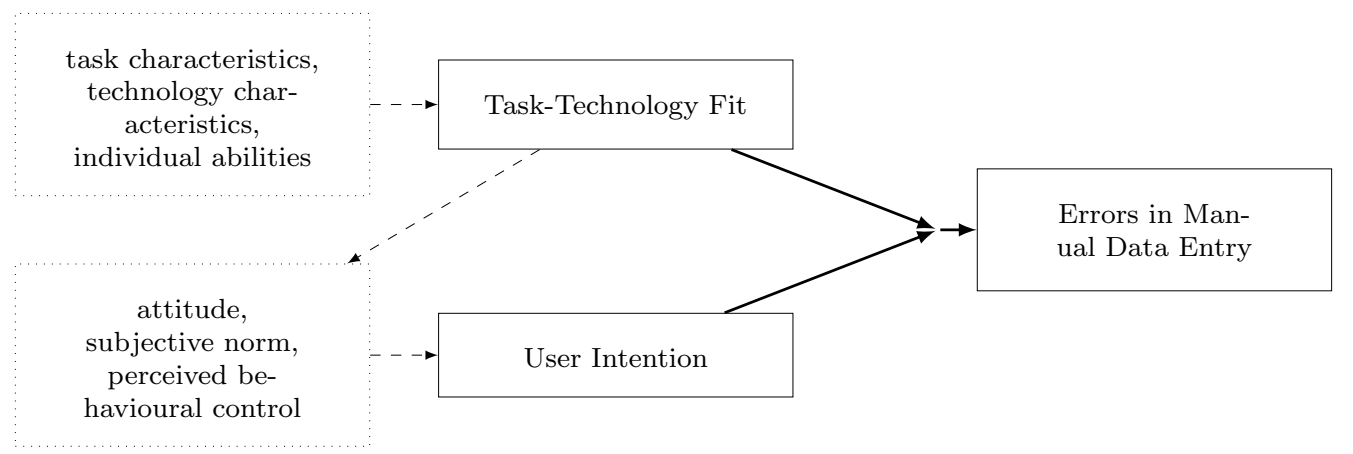

Figure 3: The CEMAD (Causes of Errors in Manually Acquired Data) framework. The core interactions of the framework are depicted in bold. The hypotheses underlying the core interactions will be tested in the remainder of the study.

Task-Technology Fit $\&$ Antecedents of Task-Technology Fit. When tailored to manual data entry, TTF is defined as 'the degree to which a technology assists the individual [data producer] in performing his or her [manual data acquisition] task' (Goodhue and Thompson, 1995, p. 216). A manual data acquisition task entails that the individual exerts a certain behaviour that turns an observation of an attribute of a real-world object into a registration in a database.

User Intention $\&$ Antecedents of User Intention. In the CEMAD framework, user intention is defined as the 'readiness' of data producers to enter data without errors (Fishbein and Ajzen, 2010, p. 43). This definition is in accordance with how intention is defined in the theory of planned behaviour (Ajzen, 1991).

The Impact of User Intention and Task-Technology Fit on Errors in Manually Acquired Data. Errors in manually acquired data are caused by a low intention of the data producers to enter high quality data and/or a low degree of TTF. A stronger intention of the users to produce high quality data or an increase in the degree of TTF will lead to fewer errors. These propositions are presented in Hypotheses 1 and 2.

Hypothesis 1. An increase in the intention of the data producers to enter correct data will cause an increase in the number of correctly entered registrations.

Hypothesis 2. An increase in the fit between the data entry task, technology and data producers will cause an increase in the number of correctly entered registrations.

At the same time, we expect an interaction effect between the degree of TTF and the intention of the data producers to enter high quality data. For example, the intention of the data producers towards entering high quality data can be reinforced by increasing the degree of TTF as it might affect the perceived behavioural control and experiential attitude of these data producers (Murphy, 2009, p. 1885). This premise is stated in Hypothesis 3.

Hypothesis 3. An increase in the fit between the data entry task, technology and data producers strengthens the effect of increasing the data producers' intention, thus increases the number of correctly entered registrations. 


\subsection{The Need for Additional Empirical Evidence}

Both theory of planned behaviour and TTF model have been tested multiple times in several empirical studies resulting in a multitude of evidence supporting their underlying hypotheses (for meta reviews see e.g. Armitage and Conner, 2001; Hardeman et al., 2002; Steinmetz et al., 2016; Cane and McCarthy, 2009). Yet, this plethora of empirical evidence is not sufficient to justify the effects of the CEMAD framework's constructs. In what follows, we explain per construct, why additional empirical evidence is required.

\subsubsection{Task-Technology Fit}

The claims concerning the TTF construct could have been supported by empirical evidence stemming from studies empirically investigating the TTF model (TTF construct), or theory of planned behaviour (actual behavioural control). However, the evidence from these studies is not sufficient for the following reasons.

On the one hand, existing TTF studies always employ the TTF construct to explain how errors in the output of an information system affect the performance of a task for which this output is used. In contrast, in the CEMAD framework, TTF is expected to explain errors in the input of an information system. This significant difference in context requires additional empirical evidence and an adaptation on how this construct should be measured.

In its original context, the degree of TTF has mostly been measured by employing user evaluations. Previous research has indicated this to be an effective measurement technique under the assumption that 'users will give evaluations based on the extent to which systems meet their needs and abilities' (Goodhue, 1995, p. 1830, emphasis added). However, in the case of manual data acquisition, the increase in performance, i.e. the reduction of the frequency or size of errors in manually entered data, will not always directly benefit the user. For example, during the interviews with the data producers, we were given the impression that these employees were positive about the technology to enter home loans, while during the observation of a data entry task, we witnessed the same technology as having a poor fit with the task of manually entering data. Therefore, in this new context, the TTF construct should be assessed objectively rather than by user evaluations.

On the other hand, evidence from studies applying the theory of planned behaviour does also not suffice to justify claims concerning the TTF construct. At first sight, this is surprising as, in the context of manual data entry, the TTF construct is conceptually equal to the actual behavioural control construct of the theory of planned behaviour. Yet, the interaction between actual behavioural control and behaviour is almost never tested in empirical applications of the theory. For example, in a recent systematic literature review of applications of the theory of planned behaviour in information system research, none of the identified studies tested this hypothesis (Authors, 2018). This can be explained by the fact that the theory of planned behaviour originates from the psychological research domain, which is primarily interested in the perceptions of individuals about behavioural control (Ajzen, 1991, p. 183). 


\subsubsection{Intention}

In contrast with the role of TTF in manual data acquisition, the effect of intention on the behaviour of individuals can already be reasonably well justified by the existing empirical evidence supporting the theory of planned behaviour. For example, meta-reviews of the theory of planned behaviour indicate that many studies reported a correlation between the intention of individuals and their behaviour (e.g. Armitage and Conner, 2001, p. 489). However, because the individuals' intention is rarely tested in combination with their degree of actual behavioural control, there are at least two interesting aspects about the effect of the data producers' intention on manually entered data that remain unclear: (1) the magnitude of the interaction between intention and TTF on the amount of errors and (2) the relative importance of both core constructs. Therefore, in the next sections, we empirically test the impact of intention versus TTF on the correctness of a data entry task.

\section{Study 1: Empirically Testing the CEMAD Framework Using an Experimental Approach}

In this part, we empirically test the hypotheses underlying the core interactions of the CEMAD framework presented in Figure 3 by means of an experiment on Amazon Mechanical Turk (MTurk).

MTurk is a platform to recruit human workers to perform Human Intelligence Tasks (HITs) in return for a small monetary reward. Typically, a HIT is a task that can be easily executed by humans, but is difficult (if not impossible) to be executed by computers (Kittur et al. 2008; Ross et al. 2010). Many of the HITs on MTurk require little time and effort (Kittur et al., 2008, p. 454) and reward human workers an amount between $0.01 \$$ and $1 \$$ (Paolacci et al., 2010). This means that MTurk enables researchers to engage a large sample of individuals to execute a simple task for a small amount of money. Not surprisingly, MTurk has been widely used to collect research data in a variety of scientific disciplines.

Because MTurk is often used to collect research data, the validity and reliability of using MTurk as a research tool are frequently investigated. In such investigations, the MTurk workers are compared against other populations, and the platform is used to replicate other influential experiments. Regarding the population of MTurk, samples of MTurk workers are found to be a better representation of the US population than samples of other panels or student samples and thus have the potential to generalise to a more varied population than traditional methods (Kittur et al., 2008). The replication studies conducted on MTurk show that results of other experiments replicate well (Crump et al., 2013; Weinberg et al., 2014), if the experimental design is simple (Krupnikov and Levine, 2014). These investigations demonstrate that MTurk is a valid research environment researchers can use to conduct experiments (Mason and Suri, 2012)

Despite its many advantages, research has pointed to several concerns that need to be considered when using the MTurk to collect research data. One frequently mentioned concern is that the environment of the participants cannot be controlled in the same way actual laboratory environments can be controlled. For example, MTurk workers might have 
installed a different browser, disabled JavaScript, experience technical problems, or experience distractions (Kittur et al., 2008). As detailed in the next section, care has been taken to design the experiment to minimise the potential effect of such elements.

MTurk has one characteristic that makes this platform particularly well-suited to empirically test the core interactions of the CEMAD framework: almost all of the HITs found on the platform can be classified as data entry tasks. For example, HITs range from transcribing text, to classifying images, translating small texts, filling in academic questionnaires, identifying objects in images, finding relevant information, processing natural language and transcribing audio (Kittur et al., 2008; Paolacci et al., 2010; Buhrmester et al., 2011). This characteristic benefits the validity of our study in two significant ways. First, by using MTurk, we can conduct an experiment that can be disguised as yet another data entry task without telling the subjects that they are participating in a data entry experiment. As such, the experiment will not have any 'demand characteristics' that could make the subjects, for example, behave in a more favourable way (Orne, 1969) such as observed in the Hawthorne studies (Roethlisberger and Dickson, 1939). Second, because many other HITs on MTurk can be classified as data entry tasks, workers are likely to be experienced in performing such tasks. This experience is comparable to the experience of workers in organisations who perform data entry tasks as part of their job. In other words, the context of an experiment on MTurk is comparable to the organisational context for which the CEMAD framework was designed.

\subsection{Method}

\subsubsection{Design \& Sample}

The core interactions of the CEMAD framework were tested using a two-by-two factorial experimental design. In such a design, two separate factors are tested by varying each factor by two levels. In our case, we tested the factors 'intention' and 'TTF' by varying the level of these factors from low to high. Table 1 displays the design of the experiment which allows for testing the individual treatments and the interaction between intention and TTF (Shadish et al., 2002).

$$
\begin{array}{ccc}
R & X_{\text {LowInt,LowTTF }} & O \\
R & X_{\text {HighInt,LowTTF }} & O \\
R & X_{\text {LowInt,HighTTF }} & O \\
R & X_{\text {HighInt,HighTTF }} & O
\end{array}
$$

Table 1: Design of the two-by-two factorial laboratory experiment. The rows represent the experimental groups and the columns represent the course of action. Letter $R$ in the first column indicates that the subjects were randomly assigned to an experimental group. Letter $X$ in the second column indicates that the subjects received a certain treatment. The treatments were designed to vary each factor by two levels corresponding to the two by two factorial design. Letter $O$ in the last column stands for the observation of the outcomes of the experiment.

In total, 2575 MTurk workers accepted the HIT containing the experiment. For every worker who accepted the HIT, we logged their worker ID and time of acceptance. Of 


\begin{tabular}{ll}
\hline Parameter & Value \\
\hline Title & 'Enter the characters as shown in the image' \\
Description & 'You will be asked to copy approximately 70 characters.' \\
Key words & 'data collection' and 'data entry' \\
Max. completion time & 30 minutes \\
Worker characteristics & Not participated in previous runs AND from UK OR US \\
Hide HIT if worker does not match the inclusion criteria? & Yes \\
Payment & $0.22 \$$ \\
Bonus & No \\
\hline
\end{tabular}

Table 2: The parameters of the MTurk HIT.

these 2575 logged workers, 2407 finished the HIT and submitted their work, resulting in an attrition rate of $6.52 \%^{2}$. One hundred and eighty-three (183) workers appeared in the log file more than once, which could indicate that these workers refreshed their page because of technical difficulties. These workers were excluded, and 2224 workers remained. Some of the remaining 2224 workers participated in previous pilot runs of the experiment and could be biased if they happened to be assigned to a different experimental group in the previous run. For example, if a worker was assigned in the first run to the intention group, and in the actual run to the control group, the worker's intention might be higher than a worker who did not yet participate. To prevent such a bias, we removed the 199 workers who participated in a previous run of the experiment. Finally, because the initial plan was to sample 2000 workers, we picked the first 2000 workers of the remaining $2025^{3}$.

\subsubsection{Procedure \& Treatment}

The experiment was disguised as a normal data entry task using a HIT on MTurk with the characteristics in Table 2.

On MTurk, before a potential worker executes a HIT, s/he can preview the task before actually accepting the HIT. In our case previewing the HIT could potentially lead to a bias. For example, a worker with a low intention to execute the HIT correctly might decide not to accept the HIT while a worker with a high intention to enter the data correctly could be more inclined to start the HIT, leading to a problem of self-selection. To this end, we disabled the HIT preview feature using JavaScript.

If a worker accepted the HIT, s/he was assigned to an experimental group in a round robin fashion based on the experimental group of the previous worker.

The actual data entry task was a request to copy a string of characters from a picture and was designed to strike a balance between clarity and difficulty. Clarity was ensured by providing simple instructions and by avoiding characters that could be misinterpreted (e.g. $\mathrm{O}$ vs 0 or I vs 1 vs 1 ). The difficulty of the data entry task needed to be ensured so it would be easier to observe an effect of the factors under investigation. Therefore, we created a string of 70 characters consisting of 'A', 'C', ' $\mathrm{G}$ ' and ' $\mathrm{T}$ ', and, to avoid copy/pasting, we presented this string using an image ${ }^{4}$. The data entry task was presented to the control

\footnotetext{
${ }^{2}$ The distribution of the attrition rate was analysed in more detail (Appendix A.1).

${ }^{3}$ Adhering to a predetermined data collection stopping rule is essential to avoid false positive results (Simmons et al., 2011).

${ }^{4}$ As will be explained, the characters were deliberately chosen to make the intention treatment more credible.
} 
group using the web page shown in Figure 4.

(This HIT will be automatically approved after one minute and you will receive your reward in any case.)

Please copy the characters from the image in the text box.

ACAAGATGCCATTGTCCCCCGGCCTCCTGCTGCTGCTGCTCTCCGGGGCCACGGCCACCGCTGCCCTGCC

Submit

Figure 4: The web page shown to the subjects in the control group.

For each treatment group, the data entry task was modified to cause an increase in the intention construct, the TTF construct or both constructs combined.

To strengthen the intention of the subjects to enter data correctly, we tried to convince them of the importance of entering the data correctly. We told them that correct data entry would benefit cancer research ${ }^{5}$ and explicitly stated that the string of characters is a DNA sequence (which is commonly represented by the characters ' $A$ ', ' $C$ ', ' $G$ ' and ' $T$ ' and explains why we chose those characters ${ }^{6}$ ). In most real-world settings, data producers are paid a fixed wage, and their income does not depend on their performance. So, to avoid the intention being influenced by monetary considerations, we explicitly stated that the HIT would be automatically approved after one minute and that the worker would receive his/her reward in any case. The resulting HIT, which was presented to the intention group, is shown in Figure 5.

(This HIT will be automatically approved after one minute and you will receive your reward in any case.)

Please copy the DNA sequence from the image in the text box. It is important that the DNA sequence is entered without errors because incorrect data will negatively affect cancer research.

ACAAGATGCCATTGTCCCCCGGCCTCCTGCTGCTGCTGCTCTCCGGGGCCACGGCCACCGCTGCCCTGCC

Submit

Figure 5: The web page shown to the subjects in the intention group.

To increase the TTF, we made the data entry task less difficult by grouping the characters in groups of 5 characters, separated by dashes. The resulting HIT, which was presented to the TTF group, is shown in Figure 6.

\footnotetext{
${ }^{5}$ We kept our promise and donated, for each correctly entered data item $0.25 \$$, to cancer research.

${ }^{6}$ We are aware that some workers in the control or TTF group might have known that they were entering a DNA sequence. Consequently, some of these workers would have a greater intention than other workers of the same group. Yet, if this would have inflicted a bias, it will have caused an underestimation of the effect of intention.
} 
(This HIT will be automatically approved after one minute and you will receive your reward in any case.)

Please copy the characters from the image in the text box.

ACAAG-ATGCC-ATTGT-CCCCC-GGCCT-CCTGC-TGCTG-CTGCT-CTCCG-GGGCC-ACGGC-CACCG-CTGCC-CTGCC

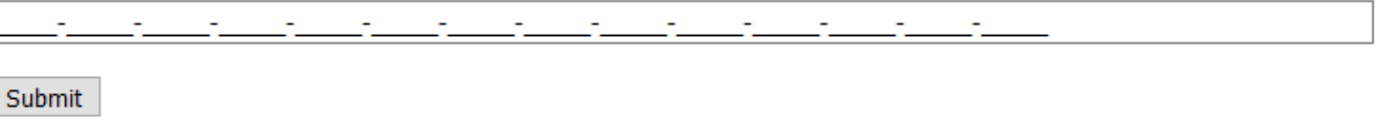

Figure 6: The web page shown to the subjects in the TTF group.

The group that received both treatments was presented the page shown in Figure 7.

(This HIT will be automatically approved after one minute and you will receive your reward in any case.)

Please copy the DNA sequence from the image in the text box. It is important that the DNA sequence is entered without errors because incorrect data will negatively affect cancer research.

ACAAG-ATGCC-ATTGT-CCCCC-GGCCT-CCTGC-TGCTG-CTGCT-CTCCG-GGGCC-ACGGC-CACCG-CTGCC-CTGCC

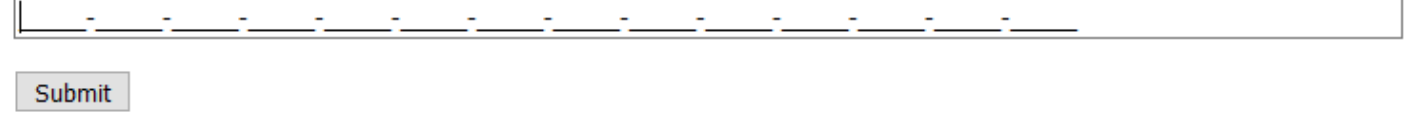

Figure 7: The web page shown to the subjects in both treatments group.

\subsubsection{Analysis}

The individual observations were analysed using a logistic regression model. Logistic regression is an established way to analyse data from a two by two factorial experiment (Montgomery et al., 2003) and only assumes that the residuals follow the binomial distribution (Peng et al., 2002). In our case, the binomial assumption is robust because the observations are randomly sampled (Peng et al., 2002).

When the logistic regression model only contains binary regressors, as in this study, its fit can be evaluated by comparing the model against the saturated model using the likelihood ratio test (Nagelkerke et al., 2005). If the likelihood ratio test indicates there is likely no difference between the residuals of the tested model and the saturated model (the perfect model), the tested model is likely to have a good fit. The likelihood ratio test comparing the fitted model to the saturated model returned $\chi^{2}=1953.4, d f=-1996, p<0.748$. This means that the null hypothesis cannot be rejected and that the fitted model is likely to be similar to the perfect model, indicating that the logistic regression model has a good fit.

\subsection{Results}

The summary of the individual responses is presented in a three-dimensional contingency table in Table 3. The logistic regression model to further analyse the individual responses is presented in Equation 1. The results of the regression analysis are shown in Table 4. 
The probabilities of entering the data correctly (marginal effects), derived from the logistic regression model are summarised in Figure 8.

\begin{tabular}{lllll}
\hline \multicolumn{2}{c}{ Factor } & \multicolumn{3}{c}{ Outcome } \\
Intention & TTF & Correct & Not Correct & Total \\
\hline \multirow{2}{*}{ Low } & Low & 294 & 207 & 501 \\
& High & 442 & 69 & 511 \\
High & Low & 353 & 117 & 470 \\
& High & 465 & 53 & 518 \\
Total & & 1554 & 446 & 2000 \\
\hline
\end{tabular}

Table 3: The three-dimensional contingency table of the individual responses.

$$
\text { IsCorrect }=\beta_{0}+\beta_{1} \text { Intention }+\beta_{2} T T F+\beta_{3}(\text { Intention } \times T T F)
$$

\begin{tabular}{lllcl}
\hline Coefficient & Estimate & Std. Error & $p$ value & \\
\hline Intercept & 0.35086 & 0.09073 & $<0.001$ & $* * *$ \\
Intention & 0.75343 & 0.14004 & $<0.001$ & $* * *$ \\
TTF & 1.50634 & 0.15807 & $<0.001$ & $* * *$ \\
Intention $\times$ TTF & -0.43889 & 0.23955 & 0.067 & $*$ \\
\hline
\end{tabular}

Table 4: The results of the logistic regression analysis. The estimates are presented on the log-odds scale and are presented with their standard error and $p$ value. Legend: significant at the $10 \% *, 5 \% * *$ and $1 \% * * *$ significance level.

The results of this study provide support for all identified hypotheses, except for the hypothesis concerning the interaction effect between intention and TTF.

The hypothesis regarding the effect of the data producers' intention is supported at the $1 \%$ significance level. The coefficient of intention in the logistic regression model is positive and has a $p$ value of less than 0.001 (see Table 4). According to the same model, as summarised in Figure 8, an increase in intention caused the data producers to enter the data more correctly: the percentage of correctly entered data rose from $58.68 \%$ to $75.11 \%$. This increase of 16.42 percentage points or $27.99 \%$ is highly significant $(p<0.001)$.

The hypothesis concerning the effect of TTF is also supported at the $1 \%$ significance level. In the results of the logistic regression model, we see that the coefficient of this factor is positive with a $p$ value of less than 0.001 . The summary of the marginal effects (Figure 8) shows that after improving the TTF, the percentage of correctly entered data rose from $58.68 \%$ to $86.50 \%$. This increase of approximately $47.40 \%$ is highly significant $(p<0.001)$.

The hypothesis about the interaction of intention and TTF is partially supported. In our study, we have not found evidence that an increase in TTF strengthens the effect of increasing the data producers' intention. This means that the whole is not greater than the sum of the parts. The coefficient of the interaction term in the logistic regression model is negative and marginally significant. Yet, we did find evidence that the percentage of correctly entered registrations would increase if both treatments were combined. The difference in the percentage of correctly entered data between the control group and the group that received both treatments is 31.09 percentage points or $52.97 \%$ and is highly significant $(p<0.001)$. The difference between the groups that received a single treatment and the group that 


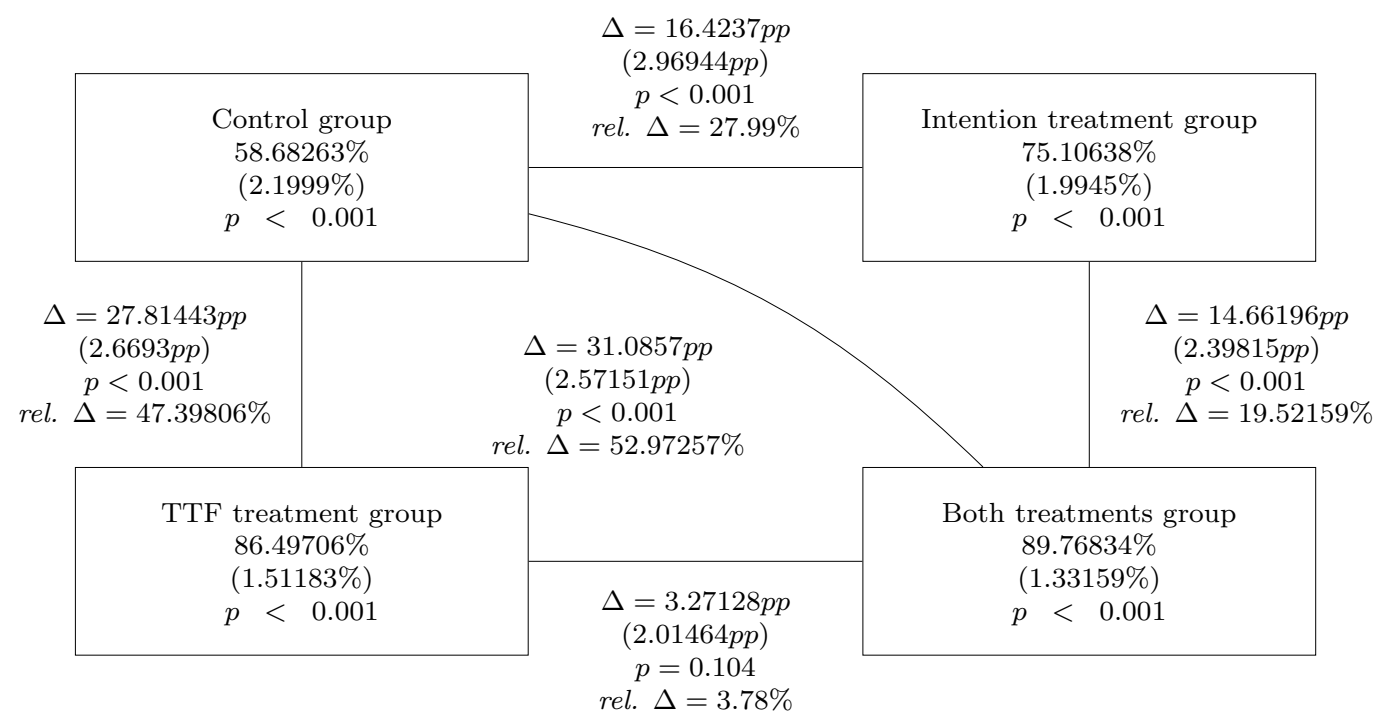

Figure 8: The marginal results per experimental group and the differences between the experimental groups including $p$ values, standard errors between brackets and relative differences.

received both treatments is 14.66 percentage points $(p<0.001)$ or $19.52 \%$ for the group that received the intention treatment and 3.27 percentage points $(p=0.104)$ or $3.78 \%$ for the group that received the TTF treatment.

Interestingly, these results show that the effect of an increase in TTF on reducing the errors in manually acquired data is more important than the effect of increasing the data producers' intention. First, as shown by the results in Table 4, the coefficient of TTF is twice as large as the coefficient of intention (log odds of 0.75 vs. 1.51). Second, as indicated by the marginal effects analysis in Figure 8, the difference between the group that received both treatments and the group that received the TTF treatment is much smaller (and only marginally significant, $p=0.104$ ) than the difference between the same group and the group that received the intention treatment (strongly significant $p<0.001$ ).

\section{Study 2: Applying the Framework in a Major Belgian Financial Institution}

The CEMAD framework was applied in a major financial institution to decrease the amount of errors in home loan data and more specifically, the errors in the value of assets that were used as collateral for these home loans. It is crucial that the value of the collateral for a home loan is correct because these home loans are sometimes used as collateral for mortgage-backed securities. The financial crisis of 2007 demonstrated that the collateral for home loans that serve as collateral for mortgage-backed securities should be correct (Acharya and Richardson, 2009).

The value of the collateral for home loans is manually entered by home loan advisers who are instructed to enter this data without error. The financial institution employs two groups of home loan advisers: senior and junior home loan advisers. The difference between 
the two is that senior home loan advisers are more experienced and are asked to disseminate knowledge and educate junior home loan advisers. In this work, we study data entry by junior home loan advisers.

While we applied the framework in the financial institution, we were given a minimum, yet highly appreciated amount of resources to study the framework's effectiveness in practice. However, due to the limited set of resources, we encountered several problems leading to important limitations of the study. For example, under perfect circumstances, to test the effect of intention on the amount of errors in the entered data, one would split the population into two groups: a treatment group and a control group, and only distribute the treatment to the treatment group. This way, one can be more confident that the effect of an increased intention was due to the intention treatment and not caused by an external factor. However, as will be explained, because the financial institution was strongly determined to prevent errors from happening, they made us distribute an additional intention treatment to all home loan advisers, and not only to a treatment group. As a consequence, the evidence of this study possesses lower degrees of internal validity compared to the MTurk study.

\subsection{Method}

\subsubsection{Design}

The application of the CEMAD framework was investigated using a study containing two components: an experimental one and an observational one.

The experimental component is depicted in Figure 5 and provides evidence concerning the effect of intention (Hypothesis 1). The experiment consisted of two behavioural interventions designed in cooperation with the company under study. The goal of these interventions was to strengthen the intention of home loan advisers to enter data correctly.

The first behavioural intervention was targeted only at half of the junior advisers and was delivered via an email containing a link to a web page showing the treatment. Because participating in the first intervention was voluntarily, the assignment of subjects to this group, called the double treatment group, was not random. As such, the experimental set-up to test this behavioural intervention is equal to a non-equivalent group design.

The second behavioural intervention was directed to all junior advisers because the company wanted to take immediate action to reduce the amount of errors in home loan data. In terms of experimental design, the approach to test the second treatment shows characteristics similar to a single group pre- and post-test design.

After the interventions were distributed, we checked the value of the collateral of two home loans for each junior home loan adviser: one that was registered before the interventions were distributed $\left(O_{1}\right)$ and one after the interventions were distributed $\left(O_{2}\right)$. This was possible because, for each asset that serves as collateral for a home loan, the home loan advisers are instructed to scan in an exhibit proving its value (e.g. sales agreement) and upload it to a digital repository. We used these exhibits to verify the value of the asset registered in the database.

In the observational component, we gathered evidence related to the TTF hypothesis. During previous studies at the same financial institution (Authors, 2018), we found that the sales agreement of the collateral for some home loans included the value of movable goods. 


$$
\begin{array}{ccccc}
N & O_{1} & X_{\text {email }} & X_{\text {peers }} & O_{2} \\
N & O_{1} & & X_{\text {peers }} & O_{2}
\end{array}
$$

Table 5: The design of the field experiment in research design notation. Half of the junior home loan advisers were randomly picked and invited via email to watch a behavioural intervention $\left(X_{\text {email }}\right)$. Some junior advisers accepted the invitation while others did not. This caused the groups to be non-equivalent (the letter $N$ stands for non-equivalent groups). Next, every senior home loan adviser was instructed to communicate to the junior advisers (i.e. their peers) that correct data entry is essential. This communication is the second behavioural intervention and was delivered to every junior adviser. Finally, we used the digital archive to check each junior adviser's record for whether s/he entered a home loan correctly before $\left(O_{1}\right)$ and after $\left(\mathrm{O}_{2}\right)$ the interventions.

If this value is mentioned on the sales agreement, the advisers should deduct this from the total amount because it is not possible to use movables as collateral for a home loan. In these cases, the task of entering the data correctly becomes more difficult and the technology does not provide help. In addition, the individual might not know which procedure s/he should follow while executing the task. Based on these considerations, whether or not the home loan includes movables can be used as a proxy to measure TTF: when such a situation occurs, the TTF is lower than usual. The TTF factor was investigated using a behavioural study because the financial institution did not allow us to make changes to the task or information system. The key downside of an observational approach compared to an experimental one is that an observational approach does not allow us to make causal inferences.

\subsubsection{Sample}

To study the application of the CEMAD framework in the financial institution, we sampled two types of entities: home loans and junior home loan advisers.

The selection of home loans was made with regard to the TTF factor. As previously explained, the TTF can vary across the sample because some home loans might be more difficult to enter than others. Here, the task becomes more complex without the technology supporting this complexity, causing the TTF between two home loans to be different. To control for the difference in TTF between home loans, we ensured that the selected home loans were either (1) equally difficult to register, or (2) that they contained an objectively observable characteristic that indicated their registration difficulty. To ensure that the home loans without an objectively observable difference in difficulty would be equally hard to enter, we only selected home loans of the most easy sort (i.e. home loans to acquire a property). As previously noted, we noticed that for some home loans the difference in difficulty was objectively observable (i.e. the sales agreement of the collateral sometimes contained movable goods). This objectively observable entering difference was used as a proxy to test the effect of TTF.

We investigated junior home loan advisers and not senior advisers because the seniors were already expected to have a strong intention to enter home loans correctly. For example, the senior advisers attend a seminar each year where they are informed about the importance of entering home loans correctly. Knowing why it is important to enter data correctly strengthens the attitude of data producers in a positive way and thus increases their intention 
to enter correct data (Authors, 2017). From the total population of approximately 750 home loan advisers, 564 are junior home loan advisers. Of these 564 junior advisers, we selected 406 junior advisers who registered a home loan before and after the interventions. Of these junior advisers who entered home loans, 202 received an email with a link to a questionnaire containing the behavioural intervention video and 110 of them filled in the questionnaire and watched the behavioural intervention video, leading to a response rate of $54.46 \%$. These 110 junior advisers who watched the video were assigned to the 'two treatments'-group. The 296 remaining advisers who did not watch the video were assigned to the single treatment group.

\subsubsection{Procedure \& Treatments}

The junior home loan advisers received one or two behavioural treatments in the form of a behavioural intervention, determined by the experimental group to which they belonged. The two interventions differ in the way they were delivered to the junior home loan advisers. The first intervention was delivered via email and the second was delivered via their peers. Both of the interventions were designed to strengthen the intention of the data producers by targeting their instrumental attitude. The advisers in the 'two treatments'-group received both behavioural interventions, and those in the single treatment group only received the behavioural intervention via their peers.

Intervention 1: Knowledge Dissemination via Email. The first intervention was delivered via email only to the 'two treatments'-group. Half of the junior home loan advisers received an email containing a link to a questionnaire containing three components. The first component contained measures regarding their current behavioural beliefs and intention about entering data correctly. These results were analysed in another study (Authors, 2018) and revealed that $31 \%$ of the data producers had a poor intention to enter data correctly. The second component, which is analysed in this study, contained the actual behavioural intervention video and a question to check whether the subjects actually watched and understood the behavioural intervention video. All the respondents answered this question correctly. The third component, which was also analysed in another study (Authors, 2017), contained measures about the effect of the behavioural intervention video on the data producers' intention, instrumental attitude, experiential attitude, subjective norm and perceived behavioural control. The results showed that the behavioural intervention video increased the intention of the data producers to enter data correctly and that this increase could be explained by an increase in their instrumental attitude (Authors, 2017). The full questionnaire and the videos are available upon request from the authors.

The behavioural intervention video, or the second component of the questionnaire, contained information on why it is important to enter the value of collateral for home loans correctly. That is, home loans are often used as collateral for mortgage-backed securities, which are a relatively cheap way for a financial institution to raise extra capital. Because errors in this data were partly responsible for the financial crisis of 2007 (Acharya and Richardson, 2009), the Belgian national bank regularly checks whether the registrations are correct. If the national bank finds too many errors, the financial institution is no longer 
allowed to sell mortgage-backed securities and has to raise capital through other, more expensive mechanisms.

All the components of the survey were constructed in close cooperation with the home loan product manager and were pre-tested in a local branch by several cognitive interviews with home loan advisers. The components were presented in Dutch, which is the mother tongue of the respondents. In addition, the video was subtitled in Dutch to ensure that every respondent would be able to receive its message, even when his/her audio was turned off.

Intervention 2: Knowledge Dissemination via Peers. The second intervention was delivered to all junior home loan advisers using the senior home loan advisers (i.e. their peers). A week after the questionnaire containing the first behavioural intervention closed, the senior advisers were invited to a seminar. During this seminar, the home loan product manager gave a presentation containing two parts. First, she explained why it is important that the value of the collateral for the home loans be entered correctly by using the same arguments that were given in the video of the first intervention. Next, she stressed that the audience of the presentation, the senior home loan advisers, should make sure this knowledge is disseminated to the junior advisers and that they could do this by showing another movie made available on the knowledge base. The presentation and the second movie are available upon request from the authors.

\subsubsection{Analysis}

The data of the field experiment, containing observations of two home loan registrations per respondent (812 observations in total), was analysed using a logistic mixed regression model. A mixed regression model is well-suited to support the hierarchical data structure resulting from the study's design.

At the lowest level, the data reflects characteristics about the home loans and at the highest level, the data reflects characteristics of the home loan advisers. In terms of fixed and random effects, the identification number of the advisers was modelled as a random effect. This random effect introduces a separate regression line per adviser and clusters the observations so each cluster contains the observations of one home loan adviser. This results in 406 clusters (i.e. junior advisers) and two observations (i.e. home loans) per cluster. The fixed effects component contains variables to model characteristics about the home loans and home loan advisers.

The fit of a mixed logistic regression model can be assessed by goodness-of-fit tests such as those proposed by Evans and Hosmer (2004), Sturdivant and Hosmer (2007) and Perera et al. (2016). However, the reliability of these goodness-of-fit tests is not investigated in cases where there is a significant number of clusters and the number of observations per cluster is low, such as in our analysis. Therefore, as an alternative, we assessed the goodness-of-fit by investigating how well the model was able to predict the outcomes of the current dataset. To this end, we calculated the $c$-statistic, which is equivalent to the area under the ROC curve. The $c$-statistic is 0.9541 and indicates that the model fits the data well. 


\begin{tabular}{lllrc}
\hline Coefficient & Estimate & Std. Error & $p$ value & \\
\hline Intercept & 2.437469 & 0.2974417 & $<0.001$ & $* * *$ \\
Adviser.InTreatmentGroup & -0.2246529 & 0.3720603 & 0.546 & $*$ \\
Loan.RegAfterTreatment & 0.5002607 & 0.287138 & 0.081 & $*$ \\
Adviser.InTreatmentGroup $\times$ Loan.RegAfterTreatment & 0.1823616 & 0.5258868 & 0.729 & $* * *$ \\
Loan.HasMovables & -3.496642 & 0.4617241 & $<0.001$ & $*$ ( \\
\hline
\end{tabular}

Table 6: The fixed effects of the mixed effects logistic regression analysis (dependent variable is Loan.IsCorrect). The identification number of the home loan adviser is included as a random effect. The estimates are presented on the log-odds scale and are presented with their standard error and $p$ value. Legend: significant at the $10 \% *, 5 \% * *$ or $1 \% * * *$ significance level.

\subsection{Results}

The result of the logistic mixed effects regression model is presented in Table 6 . The regression model contains coefficients to differentiate between the treatment and single treatment group, to make a distinction between the home loans registered before the treatment and after the treatment, and to separate home loans containing movables from those without.

Three coefficients are of particular interest. The coefficient of the interaction term (Adviser.InTreatmentGroup $\times$ Loan.RegAfterTreatment) indicates the gains of the double treatment group, relative to the single treatment group and captures the effect of the first behavioural intervention, directed at the double treatment group. Such an interaction term is often used to analyse observations resulting from a non-equivalent group design, which is then called a difference-in-difference analysis (Stock and Watson, 2012). The coefficient of Loan.RegAfterTreatment represents the effect of the second behavioural intervention directed to all junior home loan advisers. The coefficient of Loan.HasMovables reveals the (non-causal) effect of the home loan containing movables on the behaviour of the junior home loan advisers and is, as explained, a proxy for TTF.

The regression model was further studied by a marginal effects analysis (Figure 9 and 10), and the difference-in-difference analysis is graphically presented in Figure 11.

The results of these analyses provide evidence for the hypotheses concerning intention and TTF. Because we were not given any resources to change aspects of TTF (e.g. increasing the usability of the information system), the design of the study did not allow us to test the hypothesis concerning the interaction between intention and TTF.

The hypothesis about the intention of the data producers (Hypothesis 1) is weakly supported, and its evidence is mixed. On the one hand, the positive coefficient of the Loan.RegAfterTreatment parameter indicates that, all other factors zero, the behavioural intervention presented at the seminar had on average a positive effect on correctly entering home loans. This finding is marginally significant $(p=0.081)$ and provides weak support for Hypothesis 1. On the other hand, the interaction term indicating the gains of the double treatment group, relative to the single treatment group is small and not significant. Likewise, the marginal effects analyses in Figure 11 shows that for each level of Loan.HasMovables, the difference over time is small and not significant. This indicates that the effect of the behavioural intervention video is either non-existent or overshadowed by the behavioural intervention given in the seminar.

The hypothesis about the effect of TTF (Hypothesis 2) is supported. The coefficient of 


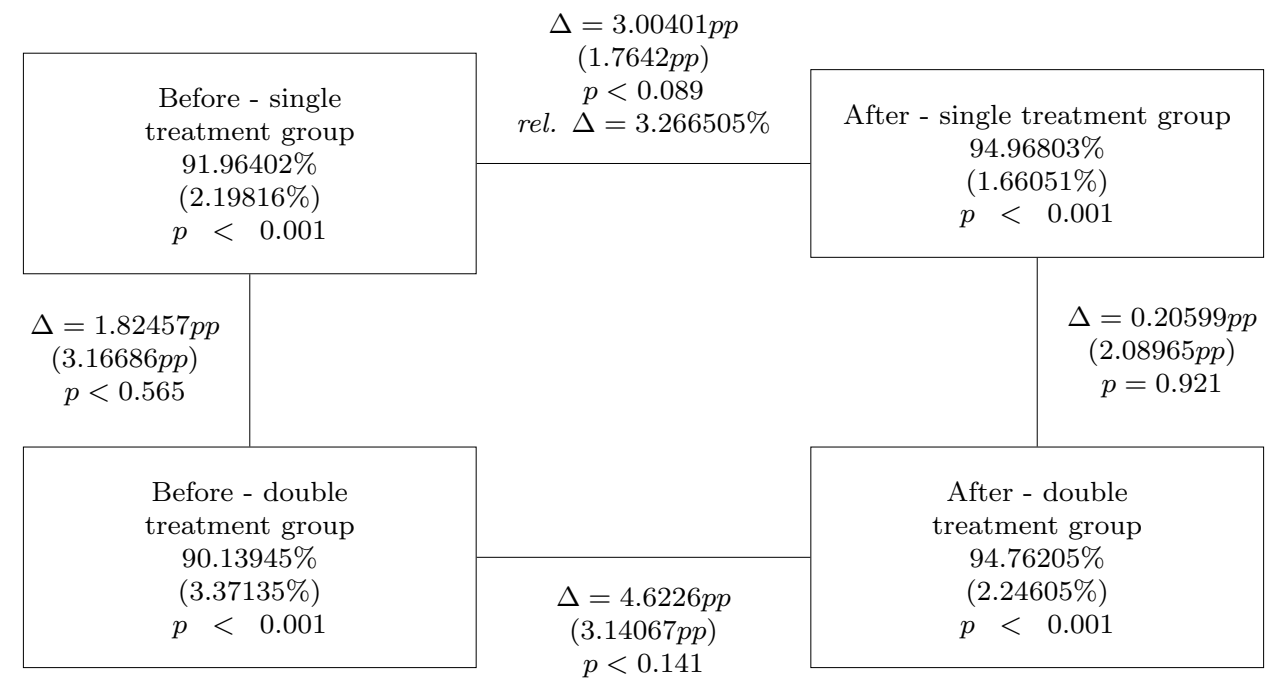

rel. $\Delta=5.128276 \%$

Figure 9: The marginal results per experimental group and the differences between the experimental groups including $p$ values, standard errors between brackets and relative differences. In this analysis, the parameter of Loan.HasMovables is set to 0.

Loan.HasMovables is large and significant at the $0.1 \%$ significance level. The marginal effects analysis further shows the impact of this factor. If the sales agreement of the collateral of the home loans did not contain movables, the percentage of correctly entered data ranges between 90 and 95 (Figure 9). Conversely, if the sales agreement contained movables, the percentage of correctly entered data ranges between 21 and 36. However, this factor was not influenced by an experimental treatment and therefore does not allow causal inference. 


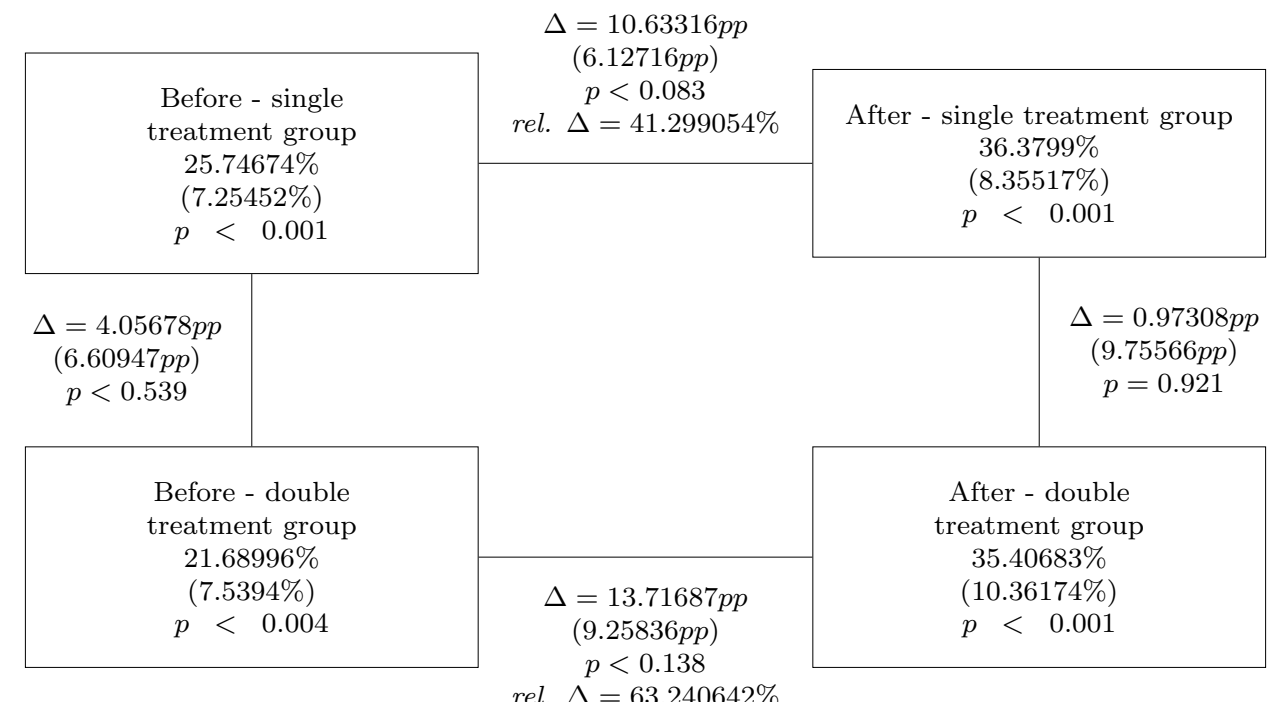

Figure 10: The marginal results per experimental group and the differences between the experimental groups including $p$ values, standard errors between brackets and relative differences. In this analysis, the parameter of Loan.HasMovables is set to 1 .
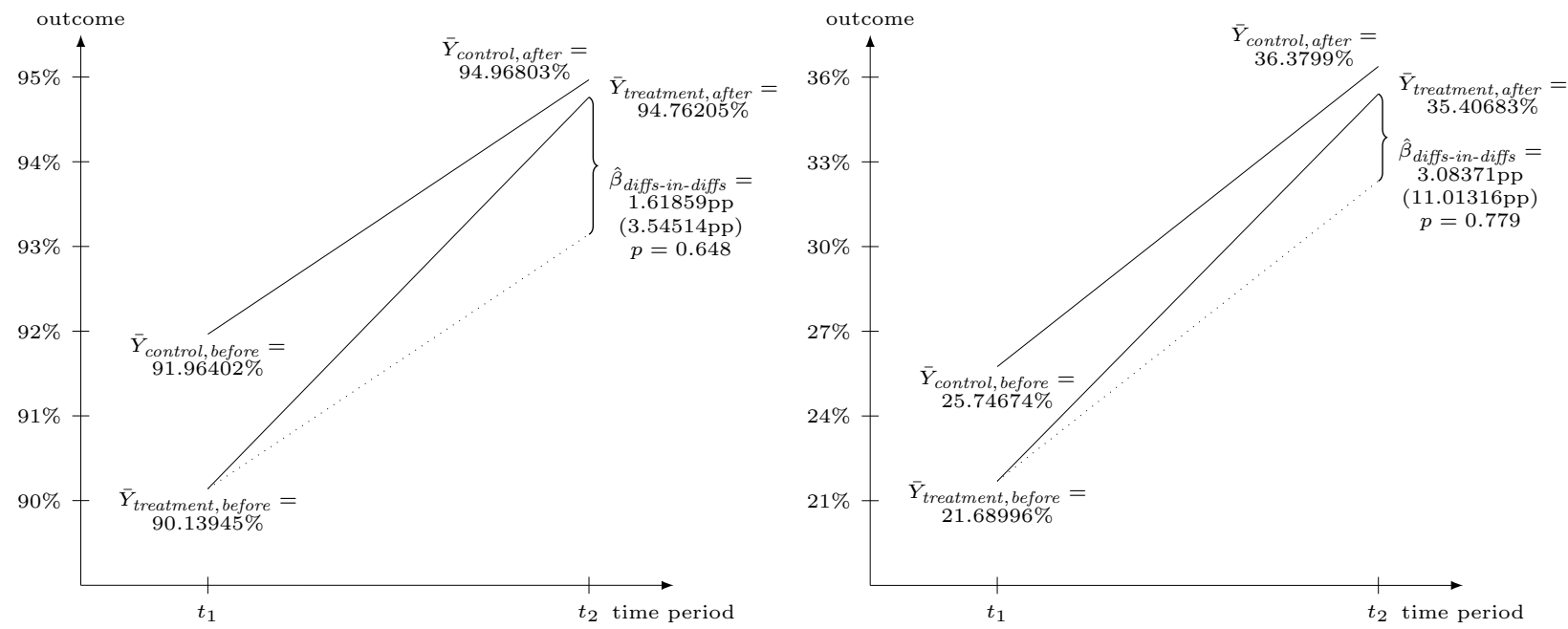

(a) In this illustration, the parameter of Loan.HasMovables is set to 0.

(b) In this illustration, the parameter of Loan.HasMovables is set to 1 .

Figure 11: Graphical illustrations of how the difference-in-difference approach was applied to investigate the gains of the double treatment group, relative to the single treatment group as a consequence of the first behavioural intervention. The outcome axis represents the error rate as estimated by the regression model. 


\section{Discussion}

Table 7 lists how the findings of the empirical studies support the hypotheses underlying the CEMAD framework. In what follows, each hypothesis is discussed in more detail.

\begin{tabular}{|c|c|c|c|}
\hline Nr. & Hypothesis & MTurk Experiment & Application Experiment \\
\hline H1 & $\begin{array}{l}\text { An increase in the intention of the data pro- } \\
\text { ducers to enter correct data will cause an } \\
\text { increase in the number of correctly entered } \\
\text { registrations. }\end{array}$ & Supported & $\begin{array}{l}\text { Weakly supported (mixed } \\
\text { evidence) }\end{array}$ \\
\hline $\mathrm{H} 2$ & $\begin{array}{l}\text { An increase in the fit between the data en- } \\
\text { try task, technology and data producers } \\
\text { will cause an increase in the number of cor- } \\
\text { rectly entered registrations. }\end{array}$ & Supported & Supported (not causally) \\
\hline H3 & $\begin{array}{l}\text { An increase in the fit between the data en- } \\
\text { try task, technology and data producers } \\
\text { strengthens the effect of increasing the data } \\
\text { producers' intention, and thus increases the } \\
\text { number of correctly entered registrations. }\end{array}$ & Partly supported & Not tested \\
\hline
\end{tabular}

Table 7: A summary of the hypotheses and how the evidence from the two empirical studies provides support for the hypotheses.

\subsection{Hypothesis 1: Intention}

The results of both experiments concerning the effect of the data producers' intention on the amount of errors in the data they entered are largely in line with our expectations. In particular, the effect of intention across the two studies has the same direction, however, the strength and the significance of the effect vary between the two studies. The results of the lab experiment exhibit a large effect of strengthening the data producers' intention on the amount of errors. For example, the free-of-error rate of the group that received the intention treatment was about $28 \%$ higher than the control group, and this effect was significant at the $1 \%$ significance level. The findings of the application of the framework in the financial institution provide mixed evidence for the intention hypothesis. To strengthen the intention of the data producers, two interventions were distributed to two groups. Both groups received the same intervention, and one group received an additional intervention. After the intervention was distributed to both groups, both groups entered the data more correctly. The size and the significance of the effect depend on which group the adviser was assigned to and whether the sales agreement of the collateral for the home loan contained movables or not. Nevertheless, the p values of these differences ranged between 0.083 and 0.141 , rendering them non-significant or only marginally significant. The intervention that was only distributed to one group had no observable effect on the amount of errors in data.

The differences between the findings of the MTurk and application experiments can be explained as follows. First, the experiment during the application of the framework suffered from sub-optimal design due to the limited amount of available resources. This sub-optimal 
design resulted in the application experiment having lower degrees of internal validity compared to the MTurk experiment. For example, the application experiment had no real control group, had a smaller sample size, its data contained fewer errors and the time between the measurements varied greatly depending on the subject. As such, the evidence of the MTurk should be treated in higher regard compared to the evidence of the application study. Second, the experimental treatment of the MTurk experiment might have had a stronger effect on the data producers' intention compared to the treatments that were given during the application of the framework in the financial institution. For example, the behavioural treatment of the MTurk experiment to increase the data producers' intention contained a statement about cancer. For some participants, this might have triggered psychosocial processes related to self-relevance, even more so when the data producers recently lost a friend or relative due to the disease. These self-relevance processes might have had a stronger effect on the data producers' intention than those triggered at the employees of the financial institution.

The fact that the behavioural intervention in the financial institution that was distributed to all home loan advisers had an observable effect and the other intervention did not is probably also related to the sub-optimal experimental design of the application study. The sub-optimal experimental design might have caused the effect of the intervention distributed to all advisers to be a false positive. That is, to observe this effect, the adopted setup was similar to a single group pre- and post-test design. The findings of such single group designs might have been influenced by external events that are unrelated to the behavioural intervention (Shadish et al., 2002). At the same time, the effect of the intervention distributed to one group of advisers might be a false negative. In a preliminary study, we found evidence that the intention of the data producers was strengthened by the behavioural intervention (Authors, 2017). The effect of their strengthened intention to enter data correctly on the amount of errors in the data these producers entered, might have been too small to observe. The free-of-error rate of the value of the collateral for the home loans was relatively high, resulting in little room for improvement or, the effect of the other behavioural intervention might have overshadowed the effect of the first behavioural intervention.

The intention of the data producers to enter data correctly can be strengthened by a behavioural intervention targeting one of the psychosocial factors as identified by the theory of planned behaviour (Authors, 2018). Which psychosocial antecedent should be targeted is contextual and should be determined on a case-by-case basis. Nevertheless, a few things should be kept in mind.

First, recent research has shown that in one data entry context, instrumental attitude was the most important determining antecedent (Authors, 2018). This can be explained as follows: in an organisational context, often, the person that enters the data is not the same as the person that will use the data (Te'eni, 1993). This situation might cause the data producer not to know why it is important to enter the data correctly, rendering the instrumental attitude of the data producers towards entering data correctly low. Thus, if the data producers are not yet aware why correct data entry is important, providing such feedback might be an inexpensive and effective way to strengthen their instrumental attitude, and as a consequence, intention towards entering data correctly (Authors, 2017). 
Second, the behavioural intervention should try to pull the data producers into entering data correctly rather than pushing them into this type of behaviour. Interventions that push data producers into entering data correctly highlight that data producers are required to enter data correctly while interventions that pull data producers into entering data correctly aim at creating an internal desire to enter data correctly. Pushing data producers into entering data correctly, for example, by increasing managerial pressure might have a small or adverse effect (Molina et al., 2013).

Third, there are many types of behavioural interventions that can be used to strengthen the intention of the data producers towards entering data correctly. Some examples are providing information, persuasion, increasing skills, goal setting, and so on (Hardeman et al., 2002). Recent research has pointed out that the most effective behavioural intervention types are motivational appeals, persuasion and increasing skills (Steinmetz et al., 2016).

\subsection{Hypothesis 2: Task-Technology Fit}

The evidence of both empirical studies strongly supported the hypothesis concerning TTF. Interestingly, in both studies, as evidenced by the coefficients of the regression models, TTF had a much greater effect on/correlation with the amount of errors in the manually acquired data than the increased intention of the data producers. This observation is similar to the observation of the study where the TTF model was proposed. That is, Goodhue and Thompson (1995) found that, compared to other factors, TTF had the greatest power in explaining the performance of individuals when utilising managerial information (Goodhue, 2006).

However, the importance of the TTF or actual behavioural constructs, which are conceptually equal in the context of manual data entry, is often neglected when behavioural theories are applied in an information system context. For example, in a recent overview of studies that empirically and quantitatively tested the theory of planned behaviour in an information system context, none of the studies included measures for actual behavioural control (Authors, 2018). In addition, several frequently cited individual information system theories such as the technology acceptance model (Davis, 1989), do not include constructs that capture the degree to which individuals have control over using information system artefacts (Goodhue, 2006).

Therefore, the findings of this study can be considered additional evidence for the relative importance of the TTF construct in the context of information systems.

\subsection{Hypothesis 3: Interaction between Task-Technology Fit and Intention}

The interaction of intention and TTF (Hypothesis 3) was only tested in the MTurk study. The evidence of the MTurk study partly supported this hypothesis. That is, the evidence showed there was most likely an interaction between both constructs, but, that the interaction of both treatments has the opposite direction as was anticipated. The effect of the combination of both treatments is not greater, but smaller than the sum of the individual treatment effects. Nevertheless, the combination of both treatments resulted in a lower amount of errors than each treatment separately. 
That the combination of treatments is not greater than the sum of parts can be explained by the law of diminishing returns. This law states that at a certain point, adding a unit of a factor that determines production output will result in a lower incremental per-unit return. In this case, error-free data can be considered as production output and the factors that determine this output are intention and TTF. Obviously, it is easier to increase an error-free rate of $50 \%$ to $51 \%$ than to increase the same rate from $98 \%$ to $99 \%$.

\section{Implications for Practice}

From a practical viewpoint, the CEMAD framework can mainly be used to guide data quality improvement actions. For example, if during data quality assessment it was found that the errors were mostly caused by a low TTF, future actions that aim to improve the quality of the assessed data should invest in closing the gap between the task requirements, abilities of the individual data producer and the functionality of the technology. During our case study, for instance, we found that there was a low fit between the data entry task and the technology because the information system required the data producers to enter the net monthly wage of the obligors, while in reality the obligors could receive a weekly wage, a variable wage or be self-employed. Because of to this poor fit between the task and the technology, the data producers had to register the data according to various and complex business rules. As a result, the wage of the obligors was sometimes erroneously entered. To reduce the errors in the wage of the obligors, we advised the organisation to redesign the information system so the structure of the data would correspond to the structure of the reality. In contrast, if a data quality assessment points into the direction of the data producers having a low intention to enter data correctly (e.g. there are many rounding errors), organisations are advised to distribute behavioural interventions aimed at increasing their intention. In some cases, both factors of the CEMAD framework should be targeted.

When organisations use the framework to reduce errors in manually acquired data, the following should be kept in mind. First, the decision on which factor(s) to improve and how this/these factor(s) should be improved should be made with the context in mind and should be based on a cost/benefit analysis. On the one hand, error-free data will be beneficial for the organisation, while on the other hand, reducing errors in data leads to a certain cost. During the application of the framework, this trade-off was especially relevant as distributing behavioural interventions was probably less effective than if we would have increased the TTF. However, in this case, and probably in other cases as well, distributing a behavioural intervention was cheaper than the alternative. Second, when interventions are applied to reduce the amount of errors in manually acquired data, new errors are prevented from entering the database, but existing errors still reside in the database. Therefore, after new errors are prevented from entering the database, organisations should resolve the existing errors, by undertaking data cleaning activities. 


\section{Conclusion \& Future Research}

The goal of this research was to propose a theoretical framework that can help organisations to explain the errors in their manually acquired data and therefore can serve as a basis to guide future data entry improvement actions.

In response, we introduced and empirically validated the CEMAD (Causes of Errors in Manually Acquired Data)-framework using several empirical studies each with a distinct research method in a total of two separate settings. The CEMAD framework states that errors during manual data entry are caused by a weak intention of the data producers to enter the data appropriately and/or by a low degree of fit between the data entry task, the technology and the data producer.

The CEMAD framework is informed by the existing theories on user behaviour and TTF but provides more insights in two ways. First, our theoretical framework refines the theory of planned behaviour with the TTF construct so it can function as a basis for concrete guidelines on data quality improvement. Second, the proposed framework demonstrates that the TTF construct, which is mostly used to explain how errors in the output of an information system affect the performance of a task, is also relevant to explain errors in the input of an information system, i.e. errors in manual data acquisition.

In both empirical studies, we found that to prevent errors in manual data entry, a high TTF is relatively more important than the data producers having a high intention to enter data correctly. This is additional empirical evidence for the importance of the TTF construct, a construct often neglected in information system research.

Future work should further investigate which aspects of TTF contribute the most to the correctness of manually acquired data and test the predictive power of the theoretical framework. 


\section{Appendix A. Supplementary Materials of the MTurk Study}

\section{Appendix A.1. Analysis of the Attrition Rate}

The workers who dropped out of the study could have caused a bias in the findings and are therefore studied in more detail. Table A.8 shows the distribution of dropouts and indicates the magnitude of the potential bias. From this table, we observe that the control and intention group had a higher attrition rate compared to the TTF, and TTF and intention group. This observation indicates a bias, but one that is consistent with the CEMAD theory and would not compromise our findings in a significant way. The fact that the control group had the second highest attrition can be explained as follows. The workers might have perceived the task as more difficult than the task given to the workers in the TTF or both treatments groups. For example, the intention group had the highest attrition rate. This can be explained because for this group, the task was the hardest and the workers knew that correct data was important. Most likely, people with a high intention to enter data correctly and who were in doubt that they could perform the data entry task, self selected and decided not to participate. This would mean that people with a lower intention or people who were not doubting themselves remained in the intention group. As such, if this would cause a bias in the result of the experiment, it would lead to an underestimation of the intention effect.

\begin{tabular}{llll}
\hline Experimental group & Total logged & Drop outs & Attrition rate \\
\hline Control & 645 & 43 & $6.67 \%$ \\
Intention & 641 & 69 & $10.76 \%$ \\
TTF & 645 & 28 & $4.34 \%$ \\
Intention and TTF & 644 & 28 & $4.34 \%$ \\
Total & 2575 & 168 & $6.52 \%$ \\
\hline
\end{tabular}

Table A.8: The experimental groups, the number of people that opened the MTurk page and were logged, and the number of drop outs. The HITs were made available in batches, this explains why some experimental groups have more or less logged users than others. 


\section{References}

Acharya, V. V., Richardson, M., 2009. Causes of the Financial Crisis. Critical Review 9 (4), 12-21.

Ajzen, I., 1991. The Theory of Planned Behavior. Orgnizational Behavior and Human Decision Processes 50, 179-211.

Armitage, C. J., Conner, M., 2001. Efficacy of the Theory of Planned Behaviour: A Meta-Analytic Review. The British Journal of Social Psychology 40, 471-499.

Arts, D. G. T., De Keizer, N. F., Scheffer, G.-J., 2002. Defining and Improving Data Quality in Medical Registries: A Literature Review, Case Study, and Generic Framework. Journal of the American Medical Informatics Association 9 (6), 600-11.

Authors, 2016. . pp. 15:1 - 15:10.

Authors, 2017. . IEEE.

Authors, 2018. .

Barchard, K. A., Pace, L. A., 2011. Preventing Human Error: The Impact of Data Entry Methods on Data Accuracy and Statistical Results. Computers in Human Behavior 27 (5), 1834-1839. URL http://dx.doi.org/10.1016/j.chb.2011.04.004

Buhrmester, M., Kwang, T., Gosling, S. D., 2011. Amazon's Mechanical Turk: A New Source of Inexpensive, Yet High-Quality, Data? Perspectives on Psychological Science 6 (1), 3-5. URL http://pps.sagepub.com/lookup/doi/10.1177/1745691610393980

Cane, S., McCarthy, R., 2009. Analyzing the Factors that Affect Information Systems Use: A TaskTechnology Fit Meta-Analysis. Journal of Computer Information Systems 50 (1), 108-123. URL http://www.iacis.org/jcis/jcis.php

Crump, M. J. C., McDonnell, J. V., Gureckis, T. M., 2013. Evaluating Amazon's Mechanical Turk as a Tool for Experimental Behavioral Research. PLoS ONE 8 (3).

Davis, F. D., 1989. Perceived Usefulness, Perceived Ease of Use, and User Acceptance of Information Technology. MIS Quarterly 13 (3), 319-340.

DeHoratius, N., Raman, A., 2008. Inventory Record Inaccuracy: An Empirical Analysis. Management Science 54 (4), $627-641$.

Dias, J., Patnaik, D., Scopa, E., van Bommel, E., 2012. Automating the Bank's Back Office. Tech. rep., McKinsey.

Espetvedt, M. N., Reksen, O., Rintakoski, S., Osterås, O., 2013. Data Quality in the Norwegian Dairy Herd Recording System: Agreement Between the National Database and Disease Recording on Farm. Journal of Dairy Science 96 (4), 2271-82.

URL http://www.ncbi.nlm.nih.gov/pubmed/23462169

Evans, S. R., Hosmer, D. W., 2004. Goodness of Fit Tests in Mixed Effects Logistic Models Characterized by Clustering. Communication in Statistics 33 (5), 1139-1155.

Fishbein, M., Ajzen, I., 2010. Predicting and Changing Behavior: The Reasoned Action Approach. Psychology Press.

Goldhill, D. R., Sumner, A., 1998. APACHE II, Data Accuracy and Outcome Prediction. Anaesthesia 53 (10), 937-943.

Goodhue, D. L., 1995. Understanding User Evaluations of Information Systems. Management Science 41 (12), 1827-1844.

Goodhue, D. L., 2006. Task-Technology Fit: A Critical (But Often Missing!) Construct in Models of Information Systems and Performance. In: Zhang, P., Galletta, D. (Eds.), Human-Computer Interaction and Management Information Systems: Foundations. M.E. Sharpe, Ch. 9, pp. $184-204$.

Goodhue, D. L., Thompson, R. L., 1995. Task-Technology Fit and Individual Performance. MIS Quarterly 19 (2), pp. 213-236.

Hardeman, W., Johnston, M., Johnston, D. W., Bonetti, D., Wareham, N. J., Kinmonth, A. L., 2002. Application of the Theory of Planned Behaviour in Behaviour Change Interventions: A Systematic Review. Psychology \& Health 17 (2), 123 - 158.

Josephs, L., sep 2016. This is how an airplane ended up at the wrong airport.

URL http://qz.com/775903 
Kittur, A., Chi, E. H., Suh, B., 2008. Crowdsourcing User Studies with Mechanical Turk. ACM Conference on Human Factors in Computing Systems, 453-456.

URL http://dl.acm.org/citation.cfm?id=1357127

Kozak, M., Krzanowski, W., Cichocka, I., Hartley, J., 2015. The Effects of Data Input Errors on Subsequent Statistical Inference. Journal of Applied Statistics 42 (9), 2030-2037.

URL http://www.tandfonline.com/doi/full/10.1080/02664763.2015.1016410

Krupnikov, Y., Levine, A. S., 2014. Cross-Sample Comparisons and External Validity. Journal of Experimental Political Science 1 (1), 59-80.

Laudon, K. C., 1986. Data Quality and Due Process in Large Interorganizational Record Systems. Communications of the ACM 29 (1), 4-11.

Lee, Y. W., Strong, D. M., 2003. Knowing-Why About Data Processes and Data Quality. Journal of Management Information Systems 20 (3), 13 - 39.

Mason, W., Suri, S., mar 2012. Conducting Behavioral Research on Amazon's Mechanical Turk. Behavior Research Methods 44 (1), 44:1-44:23.

URL http://www.ncbi.nlm.nih.gov/pubmed/21717266

Maydanchik, A., 2007. Data Quality Assessment. Technics Publications.

Molina, R., Unsworth, K., Hodkiewicz, M., Adriasola, E., 2013. Are Managerial Pressure, Technological Control and Intrinsic Motivation Effective in Improving Data Quality? Reliability Engineering \& System Safety $119,26-34$.

Montgomery, A. A., Peters, T. J., Little, P., 2003. Design, Analysis and Presentation of Factorial Randomised Controlled Trials. BMC Medical Research Methodology 3 (1), 26.

URL http://bmcmedresmethodol.biomedcentral.com/articles/10.1186/1471-2288-3-26

Moore v. R, 2016. NSWCCA 260.

URL https://www.caselaw.nsw.gov.au/decision/5834d37ce4b058596cba1abd

Murphy, G. D., 2009. Improving the Quality of Manually Acquired Data: Applying the Theory of Planned Behaviour to Data Quality. Reliability Engineering and System Safety 94 (12), 1881-1886.

Nagelkerke, N., Smits, J., le Cessie, S., van Houwelingen, H., 2005. Testing Goodness-of-fit of the Logistic Regression Model in Case-Control Studies Using Sample Reweighting. Statistics in Medicine 24 (1), 121130.

Newton, C., 2017. How a Typo Took Down S3, The Backbone of the Internet. URL https://www. theverge.com/2017/3/2/14792442/amazon-s3-outage-cause-typo-internet-server

Olsen, K. A., 2008. The $\$ 100,000$ Keying Error. Computer 41 (4).

Orne, M. T., 1969. Demand Characteristics and the Concept of Quasi-Controls. In: Artifact in Behavioral Research. Academic Press, Ch. 5, pp. 143-179.

Orr, K., 1998. Data Quality and Systems Theory. Communications of the ACM 41 (2), 66 - 71.

Paolacci, G., Chandler, J., Ipeirotis, P. G., 2010. Running Experiments on Amazon Mechanical Turk. Judgment and Decision Making 5 (5), 411-419.

URL http://repub.eur.nl/pub/31983

Peng, C.-Y. J., Lee, K. L., Ingersoll, G. M., 2002. An Introduction to Logistic Regression Analysis and Reporting. The Journal of Educational Research 96 (1), 37-41.

Perera, A. A. P. N. M., Sooriyarachchi, M. R., Wickramasuriya, S. L., 2016. A Goodness of Fit Test for the Multilevel Logistic Model. Communications in Statistics 45 (2), 643-659.

URL https://www.tandfonline.com/doi/full/10.1080/03610918.2013.868906

Roethlisberger, F. J., Dickson, W. J., 1939. Management and the Worker. Harvard University Press.

Ross, J., Irani, L., Silberman, M. S., Zaldivar, A., Tomlinson, B., 2010. Who are the Crowdworkers? Shifting Demographics in Mechanical Turk. ACM Conference on Human Factors in Computing Systems, 2863.

URL http://portal.acm.org/citation. cfm?doid=1753846.1753873

Shadish, W. R., Cook, T. D., Campbell, D. T., 2002. Experimental and Quasi-Experimental Designs for Generalized Causal Inference. Houghton Mifflin.

Sheeran, P., 2002. Intention-Behavior Relations: A Conceptual and Empirical Review. European Review of Social Psychology 12 (1), 1-36. 
Simmons, J. P., Nelson, L. D., Simonsohn, U., 2011. False-Positive Psychology: Undisclosed Flexibility in Data Collection and Analysis Allows Presenting Anything as Significant. Psychological Science 22 (11), 1359-1366.

URL http://journals.sagepub.com/doi/10.1177/0956797611417632

Steinmetz, H., Knappstein, M., Ajzen, I., Schmidt, P., Kabst, R., 2016. How Effective are Behavior Change Interventions Based on the Theory of Planned Behavior? Zeitschrift für Psychologie 224 (3), 216-233.

URL http://econtent.hogrefe.com/doi/10.1027/2151-2604/a000255

Stock, J. H., Watson, M. W., 2012. Introduction to Econometrics, 3rd Edition. Addison-Wesley.

Sturdivant, R. X., Hosmer, D. W., 2007. A Smoothed Residual Based Goodness-of-Fit Statistic for Logistic Hierarchical Regression Models. Computational Statistics and Data Analysis 51 (8), 3898-3912.

Te'eni, D., 1993. Behavioral Aspects of Data Production and Their Impact on Data Quality. Journal of Database Management $4(2), 30-38$.

Thiru, K., Hassey, A., Sullivan, F., 2003. Systematic Review of Scope and Quality of Electronic Patient Record Data in Primary Care. BMJ 326 (7398), 1070.

Wand, Y., Wang, R. Y., nov 1996. Anchoring Data Quality Dimensions in Ontological Foundations. Communications of the ACM 39 (11), 86-95.

Weinberg, J. D., Freese, J., McElhattan, D., 2014. Comparing Data Characteristics and Results of an Online Factorial Survey between a Population-Based and a Crowdsource-Recruited Sample. Sociological Science 1 (August), 292-310.

URL http://www.sociologicalscience.com/articles-vol1-19-292/

Weiskopf, N. G., Weng, C., 2013. Methods and Dimensions of Electronic Health Record Data Quality Assessment: Enabling Reuse for Clinical Research. Journal of the American Medical Informatics Association $20,144-151$. 


\section{Tom Haegemans}

Tom Haegemans is PhD candidate at the KU Leuven. He holds a Master's degree in Information Management and a Bachelor's degree in Applied Informatics. His research interests include: data quality measurement, representation of data quality measurements, causes of errors in manually acquired data, and data alignment strategies.

\section{Monique Snoeck}

Monique Snoeck is full professor at the KU Leuven, and visiting professor at the $U$ Namur. Her research focuses on enterprise modeling, requirements engineering, model-driven engineering and business process management. Her main guiding research themes are the integration of different modelling approaches into a comprehensive approach, the quality of models through formal grounding, model to code transformations and educational aspects of conceptual modelling. She has published over 80 peer-reviewed papers.

\section{Wilfried Lemahieu}

Wilfried Lemahieu is Dean of the Faculty of Economics and Business (FEB) of KU Leuven. He holds a master's degree in Business and Information Systems Engineering and a PhD in Applied Economics, both from KU Leuven. As a member of the Department of Decision Sciences and Information Management of FEB, he conducts research on big data storage, integration and analytics; data quality; business process management; and service oriented architectures. His research was published in leading journals such as International Journal of Information Management; Applied Soft Computing; and Decision Support Systems. 